\title{
Efficient Aerodynamic Shape Optimization
}

\author{
Antony Jameson* \\ Stanford Univerisity, Stanford, CA, 94305-4035, USA
}

\section{Introduction}

Since the present author first became involved in computational fluid dynamics, around 1970, the landscape has changed dramatically. At that time, panel methods had just come into use, and the world's fastest super computer, the Control data 6600, had only 131000 words of memory (about 1 megabyte). Prior to the break-through of Murman and Cole [1970], no viable algorithms for computing transonic flow with shock waves had been discovered. By 1980 the standard for super-computing was represented by the Cray 1, which achieved a performance of about 100 megaflops, but at least initially it was hard to obtain a Cray with more than 128 megabytes of memory. At the present time numerous laptops are available with processing speeds of 2-3 gigaherz, and a gigabyte of memory, well beyond the power of the Cray XMP of the mid-eighties. In fact the speed of the Intel microprocessors has increased more than one thousand fold in 17 years, between the 80386 of 1986 and the current Pentium 4. These developments were unimaginable in 1970.

There have been almost equally dramatic advances in algorithms, at least for some aerodynamic problems. Stemming in part from the pioneering work of Godunov, ${ }^{1}$ many effective shock capturing algorithms have been developed. Moreover, whereas the available methods for solving the steady state Euler equations in 1980 required 5000-10000 iterations to reach a reasonable level of convergence, and none would converge completely to machine zero, ${ }^{2}$ solutions of the Euler equations for flows around airfoils can now be obtained in 3-5 steps. ${ }^{3}$ These developments are reviewed by the author in an article for the Encyclopedia of Computational Mechanics. ${ }^{4}$ Some problems such as the prediction of transition and separation, or the formulation of universal turbulence model, remain recalcitrant. Nevertheless the combined advances in software and hardware have made it feasible to tackle problems of many orders of magnitude greater complexity than could contemplated 30 years ago.

Even at the outset, intelligent use of computational fluid dynamics (CFD) could have an important impact on design, and the present author has always recognized that the real challenge was not just to predict the flow over a give shape, but to find a superior shape, optimal accordingly to some useful criteria. In fact the author's first CFD program, Syn1 (July 1970) provided a complete solution to the inverse problem of designing an airfoil in ideal (irrotational and incompressible) flow which would produce a specified target pressure distribution. Stemming from discussions with Malcolm James at Douglas Aircraft, the method finds the conformal mapping which transforms a circle to the required airfoil. It is on extension of Lighthill's method, which is described by Thwaites, ${ }^{5}$ as an incomplete solution because it requires the target velocity to be specified in the circle plane. The input to Syn1 is the target pressure as a function of the arc length $s$. Then since the potential along the profile is

$$
\phi=\int q d s
$$

and $\phi$ is known in the circle plane, the angle $\theta$ in the circle plane can be determined as a function of $s$ by a Newton iteration. If the target pressure is not realizable, Syn1 finds the shape which produces the nearest attainable pressure distribution. Nowadays it runs on a laptop in less that $\frac{1}{100}$ second.

By the late eighties, following some early experiments by Hicks and Henne ${ }^{6}$ with the use of numerical optimization for airfoil and wing design, the time seemed ripe to tackle the general problem of aerodynamic

\footnotetext{
*Thomas V. Jones Professor of Engineering, Department of Aeronautics and Astronautics, AIAA Member.

Copyright (C) 2004 by the American Institute of Aeronautics and Astronautics, Inc. The U.S. Government has a royalty-free license to exercise all rights under the copyright claimed herein for Governmental purposes. All other rights are reserved by the copyright owner.
} 
shape optimization (ASO). After attending an ICASE workshop on flow control in February 1980; it occurred to the author that control theory offered an indirect route to ASO which could be for more efficient than the methods that had been previously tried. The author subsequently discovered that the idea of using control theory for shape optimization had also been explored by Pironneau for elliptic equations.'

Control theory for partial differential equations, where the control takes the form of boundary movement, is a natural extension of the calculus of variations, which enables the infinitely dimensional (Frechet) derivative of a cost function with respect to the shape to be determined by the solution of an adjoint equation. This gradient information can then be used to improve the shape, and the process can be repeated until the shape converges. With this approach one does not think in terms of a number of design parameters in the range of 10-100. Rather the shape is treated as a free surface, which might be represented in the discrete model by the surface mesh points, or an expansion in an appropriate set of basis functions. In the case of airfoil design one can, for example, describe the profile by the Fourier coefficients corresponding to the Laurent series which defines the conformal mapping to a circle.

The theory of control of linear PDEs is formulated in the classic work of Lions. ${ }^{8}$ The extension to nonlinear PDEs with possibly discontinuous solutions raises some difficult issues, some of which remain open. However, the author derived the necessary adjoint equations both for transonic potential flow and the Euler equations in $1988,{ }^{9}$ and developed software for airfoil design in transonic potential flow later that year. The first numerical result was published in $1989 .{ }^{10}$ A preliminary Euler adjoint code was also developed (Syn82), and support was obtained from the AFOSR to pursue the concept further. One of the issues to be explored was whether it is better to derive the adjoint PDE in continuous form from the PDE describing the flow and then discretize it, ( the "continuous adjoint" method) or to discretize the flow equations first, and then directly derive the discrete adjoint equations (the "discrete adjoint" method).

In the author's view it is important to derive the continuous adjoint equation to gain insight into the equation's properties and the appropriate boundary condition. But the appropriate discretization should certainly reflect the discretization of the flow equations. For example if one uses an upwind scheme, the adjoint discretization appears as a downwind scheme (in reality upwind for the waves in the adjoint equation which travel in the reverse direction). When a shock capturing scheme with non-linear limiters is used, the discrete adjoint approach produces very complicated discrete equations. In practice the continuous adjoint approach has proved to be very effective, but it is sometimes easier to treat the boundary conditions by the discrete approach. ${ }^{11}$

In 1993 the author coded an adjoint solver for the three-dimensional Euler equation (Syn87), and applied it to wing design, using a global mesh transformation. In his thesis, ${ }^{12}$ under the author's supervision, James Reuther developed techniques to obtain the gradient with respect to shape changes when an arbitrary mesh is subject to smooth perturbations. This decouples the adjoint method from the mesh generation technique, and enables the treatment of very complex configurations using any body fitted mesh. At the same time Syn87 was made available to NASA Ames, where it was coupled with NASA software for geometry control, and played an important role in the HSR program. ${ }^{13}$ During the last decade the adjoint method for shape optimization has become increasingly popular, and it has been successfully used in a variety of applications.

This paper focuses mainly on the continuous adjoint approach. The adjoint system of equations has a similar form to the flow equations, and hence the numerical methods developed for the flow equations ${ }^{14,15,16}$ can be re-used for the adjoint equations. While the gradient information obtained from the adjoint solution can be fed to any gradient based search procedure, it has proved very efficient in practice to make repeated small steps in a direction defined by a smoothing the gradient implicitly via a second order differential equation. This process, which guarantees the smoothness of the sequence of redesigned shapes, is equivalent to redefining the gradient in a Sobolev space, and it acts as an effective preconditioner, often yielding the optimum in 10-20 design cycles.

It is also shown that with the continuous adjoint approach (but not the discrete approach), it is possible to derive the gradient directly from the adjoint solution and the surface motion, independent of the mesh modification. This eliminates the need to evaluate volume integrals which depend on the mesh perturbation. If one wishes to obtain the pointwise gradient using an unstructured mesh, these integrals become very expensive because the propagation of the mesh deformation has to be calculated separately for the deflection of each surface mesh point. Their elimination from the gradient thus opens the way for shape optimization using unstructured mesh.

Recently wing planform parameters have been included as design variables and the Aerospace Computing Laboratory at Stanford University has successfully designed a wing which produces a specified lift with 
minimum drag, while meeting other criteria such as low structure weight, sufficient fuel volume, and stability and control. ${ }^{17}$ Based on the promising results from our wing planform optimization strategy applied to inviscid flow and from our viscous aerodynamic design techniques, ${ }^{18,19}$ we are now applying wing shape and planform optimization methods to viscous flow in order to take into account the viscous effects such as shock/boundary layer interaction, flow separation, and skin friction and eventually produce more realistic designs. ${ }^{20}$ The use of unstructured grid techniques hold considerable promise for aerodynamic design by facilitating the treatment of complex configurations without incurring a prohibitive cost and bottleneck in mesh generation. The computational feasibility of using unstructured meshes for design is essentially enabled by the use of the continuous adjoint approach and the reduced gradient formulas. ${ }^{21}$ Representative results for complete configurations are displayed in the final section.

\section{Formulation of the optimization procedure}

\section{A. Gradient Calculation}

For the class of aerodynamic optimization problems under consideration, the design space is essentially infinitely dimensional. Suppose that the performance of a system design can be measured by a cost function $I$ which depends on a function $\mathcal{F}(x)$ that describes the shape, where under a variation of the design $\delta \mathcal{F}(x)$, the variation of the cost is $\delta I$. Now suppose that $\delta I$ can be expressed to first order as

$$
\delta I=\int \mathcal{G}(x) \delta \mathcal{F}(x) d x
$$

where $\mathcal{G}(x)$ is the gradient. Then by setting

$$
\delta \mathcal{F}(x)=-\lambda \mathcal{G}(x)
$$

one obtains an improvement

$$
\delta I=-\lambda \int \mathcal{G}^{2}(x) d x
$$

unless $\mathcal{G}(x)=0$. Thus the vanishing of the gradient is a necessary condition for a local minimum.

Computing the gradient of a cost function for a complex system can be a numerically intensive task, especially if the number of design parameters is large and the cost function is an expensive evaluation. The simplest approach to optimization is to define the geometry through a set of design parameters, which may, for example, be the weights $\alpha_{i}$ applied to a set of shape functions $\mathcal{B}_{i}(x)$ so that the shape is represented as

$$
\mathcal{F}(x)=\sum \alpha_{i} \mathcal{B}_{i}(x) .
$$

Then a cost function $I$ is selected which might be the drag coefficient or the lift to drag ratio; $I$ is regarded as a function of the parameters $\alpha_{i}$. The sensitivities $\frac{\partial I}{\partial \alpha_{i}}$ may now be estimated by making a small variation $\delta \alpha_{i}$ in each design parameter in turn and recalculating the flow to obtain the change in $I$. Then

$$
\frac{\partial I}{\partial \alpha_{i}} \approx \frac{I\left(\alpha_{i}+\delta \alpha_{i}\right)-I\left(\alpha_{i}\right)}{\delta \alpha_{i}}
$$

The main disadvantage of this finite-difference approach is that the number of flow calculations needed to estimate the gradient is proportional to the number of design variables. ${ }^{22}$ Similarly, if one resorts to direct code differentiation (ADIFOR ${ }^{23,24}$ ), or complex-variable perturbations, ${ }^{25}$ the cost of determining the gradient is also directly proportional to the number of variables used to define the design.

A more cost effective technique is to compute the gradient through the solution of an adjoint problem, such as that developed in references. ${ }^{26,27,28}$ The essential idea may be summarized as follows. For flow about an arbitrary body, the aerodynamic properties that define the cost function are functions of the flowfield variables $(w)$ and the physical shape of the body, which may be represented by the function $\mathcal{F}$. Then

$$
I=I(w, \mathcal{F})
$$

and a change in $\mathcal{F}$ results in a change of the cost function

$$
\delta I=\frac{\partial I^{T}}{\partial w} \delta w+\frac{\partial I^{T}}{\partial \mathcal{F}} \delta \mathcal{F} .
$$

3 of 21 
Using a technique drawn from control theory, the governing equations of the flowfield are introduced as a constraint in such a way that the final expression for the gradient does not require reevaluation of the flowfield. In order to achieve this, $\delta w$ must be eliminated from the above equation. Suppose that the governing equation $R$, which expresses the dependence of $w$ and $\mathcal{F}$ within the flowfield domain $D$, can be written as

$$
R(w, \mathcal{F})=0 .
$$

Then $\delta w$ is determined from the equation

$$
\delta R=\left[\frac{\partial R}{\partial w}\right] \delta w+\left[\frac{\partial R}{\partial \mathcal{F}}\right] \delta \mathcal{F}=0 .
$$

Next, introducing a Lagrange multiplier $\psi$, we have

$$
\delta I=\frac{\partial I^{T}}{\partial w} \delta w+\frac{\partial I^{T}}{\partial \mathcal{F}} \delta \mathcal{F}-\psi^{T}\left(\left[\frac{\partial R}{\partial w}\right] \delta w+\left[\frac{\partial R}{\partial \mathcal{F}}\right] \delta \mathcal{F}\right) .
$$

With some rearrangement

$$
\delta I=\left(\frac{\partial I^{T}}{\partial w}-\psi^{T}\left[\frac{\partial R}{\partial w}\right]\right) \delta w+\left(\frac{\partial I^{T}}{\partial \mathcal{F}}-\psi^{T}\left[\frac{\partial R}{\partial \mathcal{F}}\right]\right) \delta \mathcal{F} .
$$

Choosing $\psi$ to satisfy the adjoint equation

$$
\left[\frac{\partial R}{\partial w}\right]^{T} \psi=\frac{\partial I^{T}}{\partial w}
$$

the term multiplying $\delta w$ can be eliminated in the variation of the cost function, and we find that

$$
\delta I=\mathcal{G} \delta \mathcal{F},
$$

where

$$
\mathcal{G}=\frac{\partial I^{T}}{\partial \mathcal{F}}-\psi^{T}\left[\frac{\partial R}{\partial \mathcal{F}}\right] .
$$

The advantage is that the variation in cost function is independent of $\delta w$, with the result that the gradient of $I$ with respect to any number of design variables can be determined without the need for additional flow-field evaluations.

In the case that (1) is a partial differential equation, the adjoint equation (3) is also a partial differential equation and appropriate boundary conditions must be determined. It turns out that the appropriate boundary conditions depend on the choice of the cost function, and may easily be derived for cost functions that involve surface-pressure integrations. Cost functions involving field integrals lead to the appearance of a source term in the adjoint equation.

The cost of solving the adjoint equation is comparable to that of solving the flow equation. Hence, the cost of obtaining the gradient is comparable to the cost of two function evaluations, regardless of the dimension of the design space.

\section{Design using the Euler Equations}

The application of control theory to aerodynamic design problems is illustrated in this section for the case of three-dimensional wing design using the compressible Euler equations as the mathematical model. The extension of the method to treat the Navier-Stokes equations is presented in references. ${ }^{29,30,31}$ It proves convenient to denote the Cartesian coordinates and velocity components by $x_{1}, x_{2}, x_{3}$ and $u_{1}, u_{2}$, $u_{3}$, and to use the convention that summation over $i=1$ to 3 is implied by a repeated index $i$. Then, the three-dimensional Euler equations may be written as

$$
\frac{\partial w}{\partial t}+\frac{\partial f_{i}}{\partial x_{i}}=0 \text { in } D
$$


where

$$
w=\left\{\begin{array}{c}
\rho \\
\rho u_{1} \\
\rho u_{2} \\
\rho u_{3} \\
\rho E
\end{array}\right\}, f_{i}=\left\{\begin{array}{c}
\rho u_{i} \\
\rho u_{i} u_{1}+p \delta_{i 1} \\
\rho u_{i} u_{2}+p \delta_{i 2} \\
\rho u_{i} u_{3}+p \delta_{i 3} \\
\rho u_{i} H
\end{array}\right\}
$$

and $\delta_{i j}$ is the Kronecker delta function. Also,

$$
p=(\gamma-1) \rho\left\{E-\frac{1}{2}\left(u_{i}^{2}\right)\right\},
$$

and

$$
\rho H=\rho E+p
$$

where $\gamma$ is the ratio of the specific heats.

In order to simplify the derivation of the adjoint equations, we map the solution to a fixed computational domain with coordinates $\xi_{1}, \xi_{2}, \xi_{3}$ where

$$
K_{i j}=\left[\frac{\partial x_{i}}{\partial \xi_{j}}\right], J=\operatorname{det}(K), K_{i j}^{-1}=\left[\frac{\partial \xi_{i}}{\partial x_{j}}\right],
$$

and

$$
S=J K^{-1}
$$

The elements of $S$ are the cofactors of $K$, and in a finite volume discretization they are just the face areas of the computational cells projected in the $x_{1}, x_{2}$, and $x_{3}$ directions. Using the permutation tensor $\epsilon_{i j k}$ we can express the elements of $S$ as

$$
S_{i j}=\frac{1}{2} \epsilon_{j p q} \epsilon_{i r s} \frac{\partial x_{p}}{\partial \xi_{r}} \frac{\partial x_{q}}{\partial \xi_{s}}
$$

Then

$$
\begin{aligned}
\frac{\partial}{\partial \xi_{i}} S_{i j} & =\frac{1}{2} \epsilon_{j p q} \epsilon_{i r s}\left(\frac{\partial^{2} x_{p}}{\partial \xi_{r} \partial \xi_{i}} \frac{\partial x_{q}}{\partial \xi_{s}}+\frac{\partial x_{p}}{\partial \xi_{r}} \frac{\partial^{2} x_{q}}{\partial \xi_{s} \partial \xi_{i}}\right) \\
& =0 .
\end{aligned}
$$

Also in the subsequent analysis of the effect of a shape variation it is useful to note that

$$
\begin{aligned}
S_{1 j} & =\epsilon_{j p q} \frac{\partial x_{p}}{\partial \xi_{2}} \frac{\partial x_{q}}{\partial \xi_{3}}, \\
S_{2 j} & =\epsilon_{j p q} \frac{\partial x_{p}}{\partial \xi_{3}} \frac{\partial x_{q}}{\partial \xi_{1}}, \\
S_{3 j} & =\epsilon_{j p q} \frac{\partial x_{p}}{\partial \xi_{1}} \frac{\partial x_{q}}{\partial \xi_{2}} .
\end{aligned}
$$

Now, multiplying equation(4) by $J$ and applying the chain rule,

$$
J \frac{\partial w}{\partial t}+R(w)=0
$$

where

$$
R(w)=S_{i j} \frac{\partial f_{j}}{\partial \xi_{i}}=\frac{\partial}{\partial \xi_{i}}\left(S_{i j} f_{j}\right),
$$

using (9). We can write the transformed fluxes in terms of the scaled contravariant velocity components

$$
U_{i}=S_{i j} u_{j}
$$




$$
F_{i}=S_{i j} f_{j}=\left[\begin{array}{c}
\rho U_{i} \\
\rho U_{i} u_{1}+S_{i 1} p \\
\rho U_{i} u_{2}+S_{i 2} p \\
\rho U_{i} u_{3}+S_{i 3} p \\
\rho U_{i} H
\end{array}\right] .
$$

For convenience, the coordinates $\xi_{i}$ describing the fixed computational domain are chosen so that each boundary conforms to a constant value of one of these coordinates. Variations in the shape then result in corresponding variations in the mapping derivatives defined by $K_{i j}$. Suppose that the performance is measured by a cost function

$$
I=\int_{\mathcal{B}} \mathcal{M}(w, S) d B_{\xi}+\int_{\mathcal{D}} \mathcal{P}(w, S) d D_{\xi},
$$

containing both boundary and field contributions where $d B_{\xi}$ and $d D_{\xi}$ are the surface and volume elements in the computational domain. In general, $\mathcal{M}$ and $\mathcal{P}$ will depend on both the flow variables $w$ and the metrics $S$ defining the computational space. The design problem is now treated as a control problem where the boundary shape represents the control function, which is chosen to minimize $I$ subject to the constraints defined by the flow equations (11). A shape change produces a variation in the flow solution $\delta w$ and the metrics $\delta S$ which in turn produce a variation in the cost function

$$
\delta I=\int_{\mathcal{B}} \delta \mathcal{M}(w, S) d B_{\xi}+\int_{\mathcal{D}} \delta \mathcal{P}(w, S) d D_{\xi}
$$

This can be split as

$$
\delta I=\delta I_{I}+\delta I_{I I},
$$

with

$$
\begin{aligned}
\delta \mathcal{M} & =\left[\mathcal{M}_{w}\right]_{I} \delta w+\delta \mathcal{M}_{I I}, \\
\delta \mathcal{P} & =\left[\mathcal{P}_{w}\right]_{I} \delta w+\delta \mathcal{P}_{I I},
\end{aligned}
$$

where we continue to use the subscripts $I$ and $I I$ to distinguish between the contributions associated with the variation of the flow solution $\delta w$ and those associated with the metric variations $\delta S$. Thus $\left[\mathcal{M}_{w}\right]_{I}$ and $\left[\mathcal{P}_{w}\right]_{I}$ represent $\frac{\partial \mathcal{M}}{\partial w}$ and $\frac{\partial \mathcal{P}}{\partial w}$ with the metrics fixed, while $\delta \mathcal{M}_{I I}$ and $\delta \mathcal{P}_{I I}$ represent the contribution of the metric variations $\delta S$ to $\delta \mathcal{M}$ and $\delta \mathcal{P}$.

In the steady state, the constraint equation (11) specifies the variation of the state vector $\delta w$ by

$$
\delta R=\frac{\partial}{\partial \xi_{i}} \delta F_{i}=0
$$

Here also, $\delta R$ and $\delta F_{i}$ can be split into contributions associated with $\delta w$ and $\delta S$ using the notation

$$
\begin{aligned}
\delta R & =\delta R_{I}+\delta R_{I I} \\
\delta F_{i} & =\left[F_{i w}\right]_{I} \delta w+\delta F_{i I I} .
\end{aligned}
$$

where

$$
\left[F_{i w}\right]_{I}=S_{i j} \frac{\partial f_{i}}{\partial w} .
$$

Multiplying by a co-state vector $\psi$, which will play an analogous role to the Lagrange multiplier introduced in equation (2), and integrating over the domain produces

$$
\int_{\mathcal{D}} \psi^{T} \frac{\partial}{\partial \xi_{i}} \delta F_{i} d \mathcal{D}_{\xi}=0
$$

Assuming that $\psi$ is differentiable, the terms with subscript $I$ may be integrated by parts to give

$$
\int_{\mathcal{B}} n_{i} \psi^{T} \delta F_{i_{I}} d \mathcal{B}_{\xi}-\int_{\mathcal{D}} \frac{\partial \psi^{T}}{\partial \xi_{i}} \delta F_{i_{I}} d \mathcal{D}_{\xi}+\int_{\mathcal{D}} \psi^{T} \delta R_{I I} d \mathcal{D}_{\xi}=0 .
$$


This equation results directly from taking the variation of the weak form of the flow equations, where $\psi$ is taken to be an arbitrary differentiable test function. Since the left hand expression equals zero, it may be subtracted from the variation in the cost function (13) to give

$$
\begin{aligned}
\delta I & =\delta I_{I I}-\int_{\mathcal{D}} \psi^{T} \delta R_{I I} d \mathcal{D}_{\xi}-\int_{\mathcal{B}}\left[\delta \mathcal{M}_{I}-n_{i} \psi^{T} \delta F_{i_{I}}\right] d \mathcal{B}_{\xi} \\
& +\int_{\mathcal{D}}\left[\delta \mathcal{P}_{I}+\frac{\partial \psi^{T}}{\partial \xi_{i}} \delta F_{i_{I}}\right] d \mathcal{D}_{\xi} .
\end{aligned}
$$

Now, since $\psi$ is an arbitrary differentiable function, it may be chosen in such a way that $\delta I$ no longer depends explicitly on the variation of the state vector $\delta w$. The gradient of the cost function can then be evaluated directly from the metric variations without having to recompute the variation $\delta w$ resulting from the perturbation of each design variable.

Comparing equations (15) and (17), the variation $\delta w$ may be eliminated from (20) by equating all field terms with subscript " $I$ " to produce a differential adjoint system governing $\psi$

$$
\frac{\partial \psi^{T}}{\partial \xi_{i}}\left[F_{i w}\right]_{I}+\left[\mathcal{P}_{w}\right]_{I}=0 \text { in } \mathcal{D} .
$$

Taking the transpose of equation (21), in the case that there is no field integral in the cost function, the inviscid adjoint equation may be written as

$$
C_{i}^{T} \frac{\partial \psi}{\partial \xi_{i}}=0 \text { in } \mathcal{D}
$$

where the inviscid Jacobian matrices in the transformed space are given by

$$
C_{i}=S_{i j} \frac{\partial f_{j}}{\partial w}
$$

The corresponding adjoint boundary condition is produced by equating the subscript " $I$ " boundary terms in equation (20) to produce

$$
n_{i} \psi^{T}\left[F_{i w}\right]_{I}=\left[\mathcal{M}_{w}\right]_{I} \quad \text { on } \mathcal{B} \text {. }
$$

The remaining terms from equation (20) then yield a simplified expression for the variation of the cost function which defines the gradient

$$
\delta I=\delta I_{I I}+\int_{\mathcal{D}} \psi^{T} \delta R_{I I} d \mathcal{D}_{\xi},
$$

which consists purely of the terms containing variations in the metrics, with the flow solution fixed. Hence an explicit formula for the gradient can be derived once the relationship between mesh perturbations and shape variations is defined.

The details of the formula for the gradient depend on the way in which the boundary shape is parameterized as a function of the design variables, and the way in which the mesh is deformed as the boundary is modified. Using the relationship between the mesh deformation and the surface modification, the field integral is reduced to a surface integral by integrating along the coordinate lines emanating from the surface. Thus the expression for $\delta I$ is finally reduced to the form

$$
\delta I=\int_{\mathcal{B}} \mathcal{G} \delta \mathcal{F} d \mathcal{B}_{\xi}
$$

where $\mathcal{F}$ represents the design variables, and $\mathcal{G}$ is the gradient, which is a function defined over the boundary surface.

The boundary conditions satisfied by the flow equations restrict the form of the left hand side of the adjoint boundary condition (23). Consequently, the boundary contribution to the cost function $\mathcal{M}$ cannot be specified arbitrarily. Instead, it must be chosen from the class of functions which allow cancellation of all terms containing $\delta w$ in the boundary integral of equation (20). On the other hand, there is no such restriction on the specification of the field contribution to the cost function $\mathcal{P}$, since these terms may always be absorbed into the adjoint field equation (21) as source terms. 
For simplicity, it will be assumed that the portion of the boundary that undergoes shape modifications is restricted to the coordinate surface $\xi_{2}=0$. Then equations (20) and (23) may be simplified by incorporating the conditions

$$
n_{1}=n_{3}=0, \quad n_{2}=1, \quad d \mathcal{B}_{\xi}=d \xi_{1} d \xi_{3},
$$

so that only the variation $\delta F_{2}$ needs to be considered at the wall boundary. The condition that there is no flow through the wall boundary at $\xi_{2}=0$ is equivalent to

$$
U_{2}=0,
$$

so that

$$
\delta U_{2}=0
$$

when the boundary shape is modified. Consequently the variation of the inviscid flux at the boundary reduces to

$$
\delta F_{2}=\delta p\left\{\begin{array}{c}
0 \\
S_{21} \\
S_{22} \\
S_{23} \\
0
\end{array}\right\}+p\left\{\begin{array}{c}
0 \\
\delta S_{21} \\
\delta S_{22} \\
\delta S_{23} \\
0
\end{array}\right\} .
$$

Since $\delta F_{2}$ depends only on the pressure, it is now clear that the performance measure on the boundary $\mathcal{M}(w, S)$ may only be a function of the pressure and metric terms. Otherwise, complete cancellation of the terms containing $\delta w$ in the boundary integral would be impossible. One may, for example, include arbitrary measures of the forces and moments in the cost function, since these are functions of the surface pressure.

In order to design a shape which will lead to a desired pressure distribution, a natural choice is to set

$$
I=\frac{1}{2} \int_{\mathcal{B}}\left(p-p_{d}\right)^{2} d S
$$

where $p_{d}$ is the desired surface pressure, and the integral is evaluated over the actual surface area. In the computational domain this is transformed to

$$
I=\frac{1}{2} \iint_{\mathcal{B}_{w}}\left(p-p_{d}\right)^{2}\left|S_{2}\right| d \xi_{1} d \xi_{3},
$$

where the quantity

$$
\left|S_{2}\right|=\sqrt{S_{2 j} S_{2 j}}
$$

denotes the face area corresponding to a unit element of face area in the computational domain. Now, to cancel the dependence of the boundary integral on $\delta p$, the adjoint boundary condition reduces to

$$
\psi_{j} n_{j}=p-p_{d}
$$

where $n_{j}$ are the components of the surface normal

$$
n_{j}=\frac{S_{2 j}}{\left|S_{2}\right|} .
$$

This amounts to a transpiration boundary condition on the co-state variables corresponding to the momentum components. Note that it imposes no restriction on the tangential component of $\psi$ at the boundary.

We find finally that

$$
\begin{aligned}
& \delta I=-\int_{\mathcal{D}} \frac{\partial \psi^{T}}{\partial \xi_{i}} \delta S_{i j} f_{j} d \mathcal{D} \\
& -\iint_{B_{W}}\left(\delta S_{21} \psi_{2}+\delta S_{22} \psi_{3}+\delta S_{23} \psi_{4}\right) p d \xi_{1} d \xi_{3} .
\end{aligned}
$$


Here the expression for the cost variation depends on the mesh variations throughout the domain which appear in the field integral. However, the true gradient for a shape variation should not depend on the way in which the mesh is deformed, but only on the true flow solution. In the next section we show how the field integral can be eliminated to produce a reduced gradient formula which depends only on the boundary movement.

\section{The Reduced gradient formulation}

Consider the case of a mesh variation with a fixed boundary. Then,

$$
\delta I=0
$$

but there is a variation in the transformed flux,

$$
\delta F_{i}=C_{i} \delta w+\delta S_{i j} f_{j}
$$

Here the true solution is unchanged. Thus, the variation $\delta w$ is due to the mesh movement $\delta x$ at each mesh point. Therefore

$$
\delta w=\nabla w \cdot \delta x=\frac{\partial w}{\partial x_{j}} \delta x_{j}\left(=\delta w^{*}\right)
$$

and since

$$
\frac{\partial}{\partial \xi_{i}} \delta F_{i}=0
$$

it follows that

$$
\frac{\partial}{\partial \xi_{i}}\left(\delta S_{i j} f_{j}\right)=-\frac{\partial}{\partial \xi_{i}}\left(C_{i} \delta w^{*}\right)
$$

It is verified below that this relation holds in the general case with boundary movement. Now

$$
\begin{aligned}
\int_{\mathcal{D}} \phi^{T} \delta R d \mathcal{D} & =\int_{\mathcal{D}} \phi^{T} \frac{\partial}{\partial \xi_{i}} C_{i}\left(\delta w-\delta w^{*}\right) d \mathcal{D} \\
& =\int_{\mathcal{B}} \phi^{T} C_{i}\left(\delta w-\delta w^{*}\right) d \mathcal{B} \\
& -\int_{\mathcal{D}} \frac{\partial \phi^{T}}{\partial \xi_{i}} C_{i}\left(\delta w-\delta w^{*}\right) d \mathcal{D} .
\end{aligned}
$$

Here on the wall boundary

$$
C_{2} \delta w=\delta F_{2}-\delta S_{2 j} f_{j}
$$

Thus, by choosing $\phi$ to satisfy the adjoint equation (22) and the adjoint boundary condition (23), we reduce the cost variation to a boundary integral which depends only on the surface displacement:

$$
\begin{aligned}
& \delta I=\int_{\mathcal{B}_{\mathcal{W}}} \psi^{T}\left(\delta S_{2 j} f_{j}+C_{2} \delta w^{*}\right) d \xi_{1} d \xi_{3} \\
& -\iint_{B_{W}}\left(\delta S_{21} \psi_{2}+\delta S_{22} \psi_{3}+\delta S_{23} \psi_{4}\right) p d \xi_{1} d \xi_{3} .
\end{aligned}
$$

For completeness the general derivation of equation(28) is presented here. Using the formula(8), and the property (9)

$$
\begin{aligned}
& \frac{\partial}{\partial \xi_{i}}\left(\delta S_{i j} f_{j}\right) \\
& =\frac{1}{2} \frac{\partial}{\partial \xi_{i}}\left\{\epsilon_{j p q} \epsilon_{i r s}\left(\frac{\partial \delta x_{p}}{\partial \xi_{r}} \frac{\partial x_{q}}{\partial \xi_{s}}+\frac{\partial x_{p}}{\partial \xi_{r}} \frac{\partial \delta x_{q}}{\partial \xi_{s}}\right) f_{j}\right\} \\
& =\frac{1}{2} \epsilon_{j p q} \epsilon_{i r s}\left(\frac{\partial \delta x_{p}}{\partial \xi_{r}} \frac{\partial x_{q}}{\partial \xi_{s}}+\frac{\partial x_{p}}{\partial \xi_{r}} \frac{\partial \delta x_{q}}{\partial \xi_{s}}\right) \frac{\partial f_{j}}{\partial \xi_{i}} \\
& =\frac{1}{2} \epsilon_{j p q} \epsilon_{i r s}\left\{\frac{\partial}{\partial \xi_{r}}\left(\delta x_{p} \frac{\partial x_{q}}{\partial \xi_{s}} \frac{\partial f_{j}}{\partial \xi_{i}}\right)\right\}
\end{aligned}
$$

9 of 21 


$$
\begin{aligned}
& +\frac{1}{2} \epsilon_{j p q} \epsilon_{i r s}\left\{\frac{\partial}{\partial \xi_{s}}\left(\delta x_{q} \frac{\partial x_{p}}{\partial \xi_{r}} \frac{\partial f_{j}}{\partial \xi_{i}}\right)\right\} \\
& =\frac{\partial}{\partial \xi_{r}}\left(\delta x_{p} \epsilon_{p q j} \epsilon_{r s i} \frac{\partial x_{q}}{\partial \xi_{s}} \frac{\partial f_{j}}{\partial \xi_{i}}\right) .
\end{aligned}
$$

Now express $\delta x_{p}$ in terms of a shift in the original computational coordinates

$$
\delta x_{p}=\frac{\partial x_{p}}{\partial \xi_{k}} \delta \xi_{k} .
$$

Then we obtain

$$
\frac{\partial}{\partial \xi_{i}}\left(\delta S_{i j} f_{j}\right)=\frac{\partial}{\partial \xi_{r}}\left(\epsilon_{p q j} \epsilon_{r s i} \frac{\partial x_{p}}{\partial \xi_{k}} \frac{\partial x_{q}}{\partial \xi_{s}} \frac{\partial f_{j}}{\partial \xi_{i}} \delta \xi_{k}\right) .
$$

The term in $\frac{\partial}{\partial \xi_{1}}$ is

$$
\epsilon_{123} \epsilon_{p q j} \frac{\partial x_{p}}{\partial \xi_{k}}\left(\frac{\partial x_{q}}{\partial \xi_{2}} \frac{\partial f_{j}}{\partial \xi_{3}}-\frac{\partial x_{q}}{\partial \xi_{3}} \frac{\partial f_{j}}{\partial \xi_{2}}\right) \delta \xi_{k} .
$$

Here the term multiplying $\delta \xi_{1}$ is

$$
\epsilon_{j p q}\left(\frac{\partial x_{p}}{\partial \xi_{1}} \frac{\partial x_{q}}{\partial \xi_{2}} \frac{\partial f_{j}}{\partial \xi_{3}}-\frac{\partial x_{p}}{\partial \xi_{1}} \frac{\partial x_{q}}{\partial \xi_{3}} \frac{\partial f_{j}}{\partial \xi_{2}}\right) .
$$

According to the formulas(10) this may be recognized as

$$
S_{2 j} \frac{\partial f_{1}}{\partial \xi_{2}}+S_{3 j} \frac{\partial f_{1}}{\partial \xi_{3}}
$$

or, using the quasi-linear form(12) of the equation for steady flow, as

$$
-S_{1 j} \frac{\partial f_{1}}{\partial \xi_{1}}
$$

The terms multiplying $\delta \xi_{2}$ and $\delta \xi_{3}$ are

$$
\epsilon_{j p q}\left(\frac{\partial x_{p}}{\partial \xi_{2}} \frac{\partial x_{q}}{\partial \xi_{2}} \frac{\partial f_{j}}{\partial \xi_{3}}-\frac{\partial x_{p}}{\partial \xi_{2}} \frac{\partial x_{q}}{\partial \xi_{3}} \frac{\partial f_{j}}{\partial \xi_{2}}\right)=-S_{1 j} \frac{\partial f_{1}}{\partial \xi_{2}}
$$

and

$$
\epsilon_{j p q}\left(\frac{\partial x_{p}}{\partial \xi_{3}} \frac{\partial x_{q}}{\partial \xi_{2}} \frac{\partial f_{j}}{\partial \xi_{3}}-\frac{\partial x_{p}}{\partial \xi_{3}} \frac{\partial x_{q}}{\partial \xi_{3}} \frac{\partial f_{j}}{\partial \xi_{2}}\right)=-S_{1 j} \frac{\partial f_{1}}{\partial \xi_{3}} .
$$

Thus the term in $\frac{\partial}{\partial \xi_{1}}$ is reduced to

$$
-\frac{\partial}{\partial \xi_{1}}\left(S_{1 j} \frac{\partial f_{1}}{\partial \xi_{k}} \delta \xi_{k}\right) .
$$

Finally, with similar reductions of the terms in $\frac{\partial}{\partial \xi_{2}}$ and $\frac{\partial}{\partial \xi_{3}}$, we obtain

$$
\frac{\partial}{\partial \xi_{i}}\left(\delta S_{i j} f_{j}\right)=-\frac{\partial}{\partial \xi_{i}}\left(S_{i j} \frac{\partial f_{j}}{\partial \xi_{k}} \delta \xi_{k}\right)=-\frac{\partial}{\partial \xi_{i}}\left(C_{i} \delta w^{*}\right)
$$

as was to be proved.

\section{Optimization Procedure}

\section{A. The Need for a Sobolev Inner Product in the Definition of the Gradient}

Another key issue for successful implementation of the continuous adjoint method is the choice of an appropriate inner product for the definition of the gradient. It turns out that there is an enormous benefit from the use of a modified Sobolev gradient, which enables the generation of a sequence of smooth shapes. This can be illustrated by considering the simplest case of a problem in the calculus of variations. 
Suppose that we wish to find the path $y(x)$ which minimizes

$$
I=\int_{a}^{b} F\left(y, y^{\prime}\right) d x
$$

with fixed end points $y(a)$ and $y(b)$. Under a variation $\delta y(x)$,

$$
\begin{aligned}
\delta I & =\int_{a}^{b}\left(\frac{\partial F}{\partial y} \delta y+\frac{\partial F}{\partial y^{\prime}} \delta y^{\prime}\right) d x \\
& =\int_{a}^{b}\left(\frac{\partial F}{\partial y}-\frac{d}{d x} \frac{\partial F}{\partial y^{\prime}}\right) \delta y d x
\end{aligned}
$$

Thus defining the gradient as

$$
g=\frac{\partial F}{\partial y}-\frac{d}{d x} \frac{\partial F}{\partial y^{\prime}}
$$

and the inner product as

$$
(u, v)=\int_{a}^{b} u v d x
$$

we find that

$$
\delta I=(g, \delta y) .
$$

If we now set

$$
\delta y=-\lambda g, \quad \lambda>0
$$

we obtain a improvement

$$
\delta I=-\lambda(g, g) \leq 0
$$

unless $g=0$, the necessary condition for a minimum.

Note that $g$ is a function of $y, y^{\prime}, y^{\prime \prime}$,

$$
g=g\left(y, y^{\prime}, y^{\prime \prime}\right)
$$

In the well known case of the Brachistrone problem, for example, which calls for the determination of the path of quickest descent between two laterally separated points when a particle falls under gravity,

$$
F\left(y, y^{\prime}\right)=\sqrt{\frac{1+y^{\prime 2}}{y}}
$$

and

$$
g=-\frac{1+y^{\prime 2}+2 y y^{\prime \prime}}{2\left(y\left(1+y^{\prime 2}\right)\right)^{3 / 2}}
$$

It can be seen that each step

$$
y^{n+1}=y^{n}-\lambda^{n} g^{n}
$$

reduces the smoothness of $y$ by two classes. Thus the computed trajectory becomes less and less smooth, leading to instability.

In order to prevent this we can introduce a weighted Sobolev inner product ${ }^{32}$

$$
\langle u, v\rangle=\int\left(u v+\epsilon u^{\prime} v^{\prime}\right) d x
$$


where $\epsilon$ is a parameter that controls the weight of the derivatives. We now define a gradient $\bar{g}$ such that

$$
\delta I=\langle\bar{g}, \delta y\rangle
$$

Then we have

$$
\begin{aligned}
\delta I & =\int\left(\bar{g} \delta y+\epsilon \bar{g}^{\prime} \delta y^{\prime}\right) d x \\
& =\int\left(\bar{g}-\frac{\partial}{\partial x} \epsilon \frac{\partial \bar{g}}{\partial x}\right) \delta y d x \\
& =(g, \delta y)
\end{aligned}
$$

where

$$
\bar{g}-\frac{\partial}{\partial x} \epsilon \frac{\partial \bar{g}}{\partial x}=g
$$

and $\bar{g}=0$ at the end points. Thus $\bar{g}$ can be obtained from $g$ by a smoothing equation. Now the step

$$
y^{n+1}=y^{n}-\lambda^{n} \bar{g}^{n}
$$

gives an improvement

$$
\delta I=-\lambda^{n}\left\langle\bar{g}^{n}, \bar{g}^{n}\right\rangle
$$

but $y^{n+1}$ has the same smoothness as $y^{n}$, resulting in a stable process.

\section{B. Sobolev Gradient for Shape Optimization}

In applying control theory to aerodynamic shape optimization, the use of a Sobolev gradient is equally important for the preservation of the smoothness class of the redesigned surface. Accordingly, using the weighted Sobolev inner product defined above, we define a modified gradient $\overline{\mathcal{G}}$ such that

$$
\delta I=<\overline{\mathcal{G}}, \delta \mathcal{F}>.
$$

In the one dimensional case $\overline{\mathcal{G}}$ is obtained by solving the smoothing equation

$$
\overline{\mathcal{G}}-\frac{\partial}{\partial \xi_{1}} \epsilon \frac{\partial}{\partial \xi_{1}} \overline{\mathcal{G}}=\mathcal{G} .
$$

In the multi-dimensional case the smoothing is applied in product form. Finally we set

$$
\delta \mathcal{F}=-\lambda \overline{\mathcal{G}}
$$

with the result that

$$
\delta I=-\lambda<\overline{\mathcal{G}}, \overline{\mathcal{G}}>\quad<0,
$$

unless $\overline{\mathcal{G}}=0$, and correspondingly $\mathcal{G}=0$.

When second-order central differencing is applied to (34), the equation at a given node, $i$, can be expressed as

$$
\overline{\mathcal{G}}_{i}-\epsilon\left(\overline{\mathcal{G}}_{i+1}-2 \overline{\mathcal{G}}_{i}+\overline{\mathcal{G}}_{i-1}\right)=\mathcal{G}_{i}, \quad 1 \leq i \leq n,
$$

where $\mathcal{G}_{i}$ and $\overline{\mathcal{G}}_{i}$ are the point gradients at node $i$ before and after the smoothing respectively, and $n$ is the number of design variables equal to the number of mesh points in this case. Then,

$$
\overline{\mathcal{G}}=A \mathcal{G},
$$

where $\mathrm{A}$ is the $n \times n$ tri-diagonal matrix such that

$$
A^{-1}=\left[\begin{array}{ccccc}
1+2 \epsilon & -\epsilon & 0 & . & 0 \\
\epsilon & \cdot & \cdot & & \\
0 & \cdot & \cdot & \cdot & \\
. & & \cdot & \cdot & -\epsilon \\
0 & & & \epsilon & 1+2 \epsilon
\end{array}\right] .
$$


Using the steepest descent method in each design iteration, a step, $\delta \mathcal{F}$, is taken such that

$$
\delta \mathcal{F}=-\lambda A \mathcal{G} .
$$

As can be seen from the form of this expression, implicit smoothing may be regarded as a preconditioner which allows the use of much larger steps for the search procedure and leads to a large reduction in the number of design iterations needed for convergence.

\section{Outline of the Design Procedure}

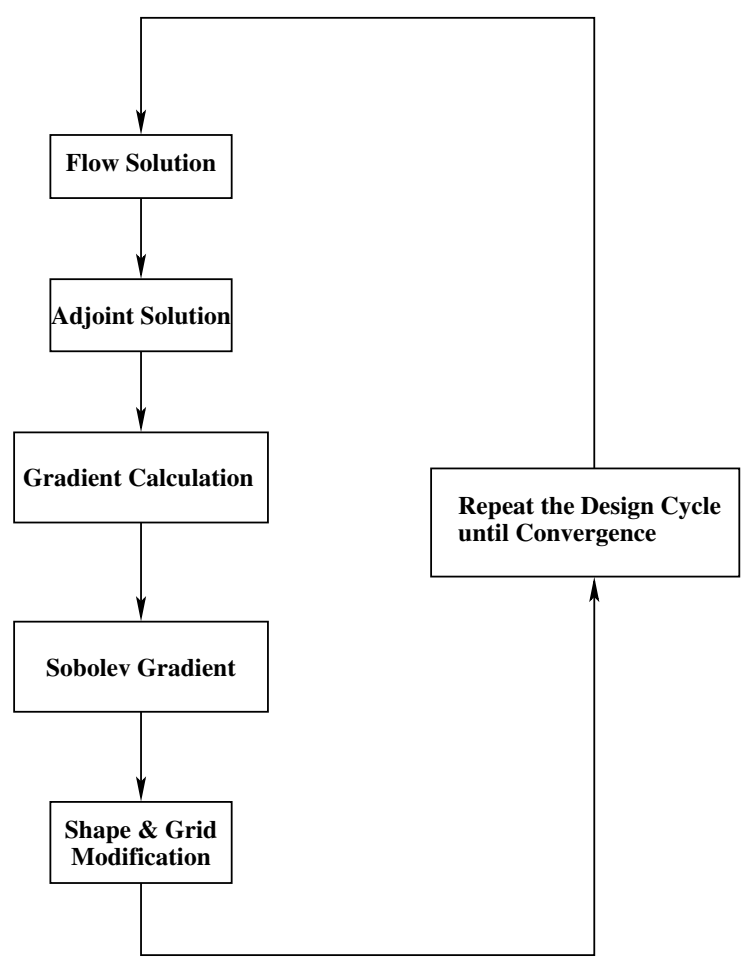

Figure 1. Design cycle

The design procedure can finally be summarized as follows:

1. Solve the flow equations for $\rho, u_{1}, u_{2}, u_{3}, p$.

2. Solve the adjoint equations for $\psi$ subject to appropriate boundary conditions.

3. Evaluate $\mathcal{G}$ and calculate the corresponding Sobolev gradient $\overline{\mathcal{G}}$.

4. Project $\overline{\mathcal{G}}$ into an allowable subspace that satisfies any geometric constraints.

5. Update the shape based on the direction of steepest descent.

6. Return to 1 until convergence is reached.

Practical implementation of the design method relies heavily upon fast and accurate solvers for both the state $(w)$ and co-state $(\psi)$ systems. The result obtained in Section VI have been obtained using wellvalidated software for the solution of the Euler and Navier-Stokes equations developed over the course of many years. ${ }^{14,33,34}$ For inverse design the lift is fixed by the target pressure. In drag minimization it is also appropriate to fix the lift coefficient, because the induced drag is a major fraction of the total drag, and this could be reduced simply by reducing the lift. Therefore the angle of attack is adjusted during each flow solution to force a specified lift coefficient to be attained, and the influence of variations of the angle of attack is included in the calculation of the gradient. The vortex drag also depends on the span loading, 
which may be constrained by other considerations such as structural loading or buffet onset. Consequently, the option is provided to force the span loading by adjusting the twist distribution as well as the angle of attack during the flow solution.

\section{Case studies}

\section{A. Two dimensional studies of transonic airfoil design}

When the inviscid Euler equations are used to model the flow, the source of drag is the wage-drag due to shock waves. Accordingly, if the shape is optimized for minimum drag at fixed lift, the best attainable result is a shock-free airfoil with zero drag. By this criterion the optimum shape is completely non-unique, since all shock-free profiles are equally good. The author's experience during the last 15 years has confirmed that shock-free profiles can be obtained from a wide variety of initial shape, while maintaining a fixed lift coefficient and a fixed thickness.

Recently the author's two-dimensional Euler design code Syn83 has been used to explore the attainable limits of Mach numbers and lift coefficient under which shock-free airfoils of a give thickness can be attained. ${ }^{35}$ When the design objectives are two extreme the performance tends to degrade very rapidly off the design point, with strong double shocks typically appearing below the design point. Thus the boundary of shockfree airfoil in the $C_{l}$-Mach space is somewhat fuzzy. The study confirms, however, that for ten-percent thick airfoils one can attain benign shock-free shapes along a boundary passing through $C_{l} .6$ and Mach .78 and $C_{l} .7$ and Mach .77. The second of these points is illustrated in figure 3. The boundary is shifted up as the thickness is reduced. In fact the transonic similarity rule can be used to find progressively thinner profiles which are shock-free at increasing Mach number, as illustrated in figure 4.

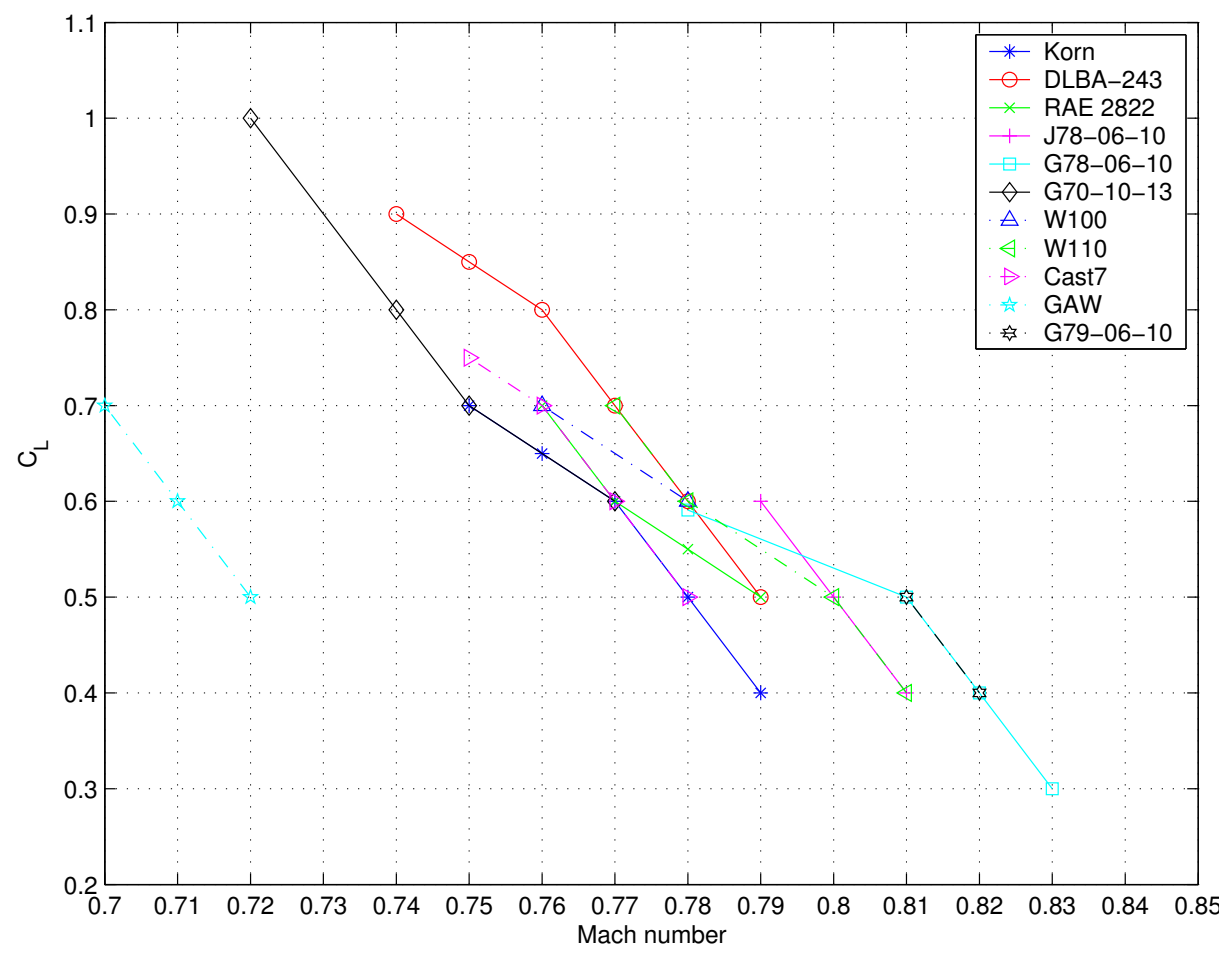

Figure 2. Attainable shock-free solutions for various shape optimized airfoils

Moreover, shock-free flow can be attained with profiles that have no resemblance to the typical flat-topped and aft-loaded super-critical section. It appears, however, that aft-loading, perhaps aided by a divergent trailing edge, can help to extend shock-free flow to higher lift coefficients. 


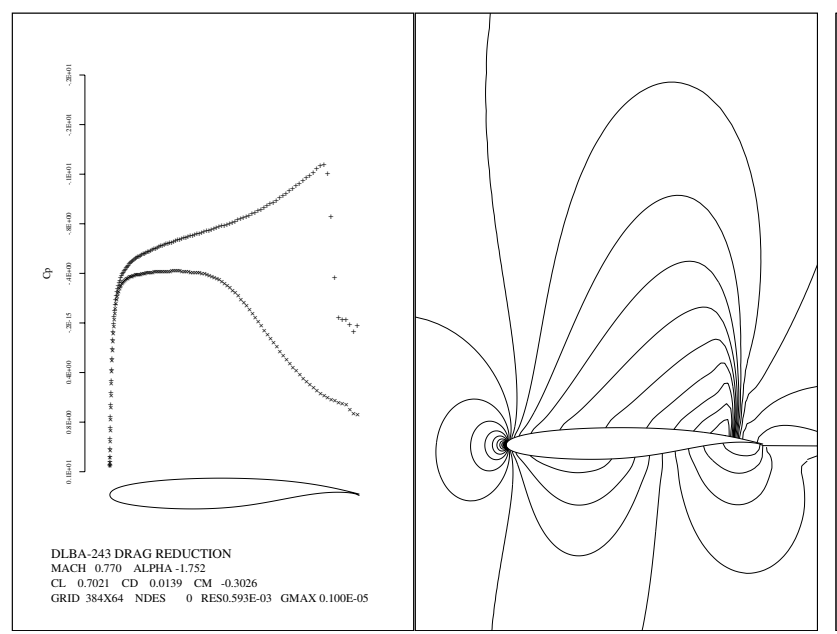

(a) Before optimization

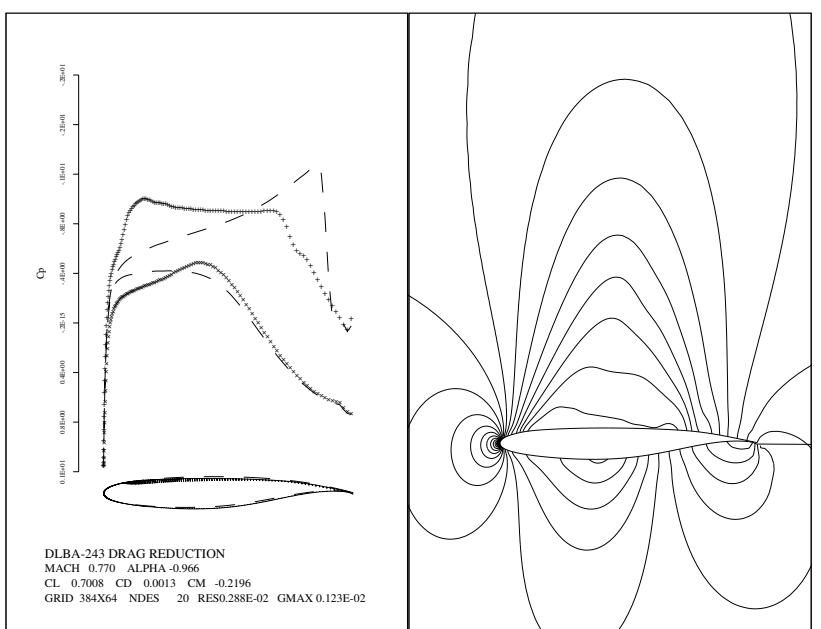

(b) After optimization

Figure 3. Pressure distribution and Mach contours for the DLBA-243 airfoil

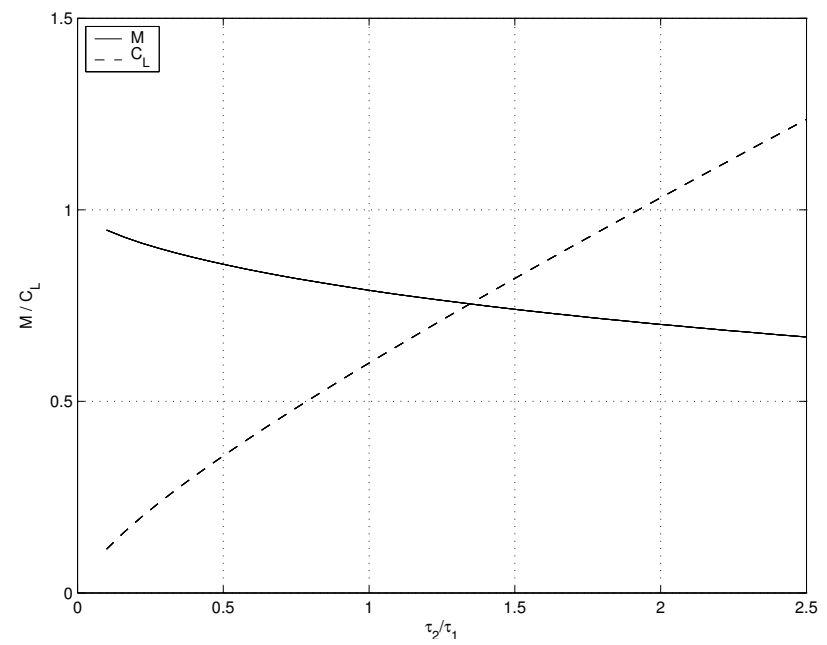

Figure 4. Transonic similarity rule: $\mathrm{M}$ and $C_{L}$ scale with thickness ratio

\section{B. B747 Euler planform result}

The shape changes in the section needed to improve the transonic wing design are quite small. However, in order to obtain a true optimum design larger scale changes such as changes in the wing planform (sweepback, span, chord, section thickness, and taper) should be considered. Because these directly affect the structure weight, a meaningful result can only be obtained by considering a cost function that accounts for both the aerodynamic characteristics and the weight.

In references ${ }^{17,20,36}$ the cost function is defined as

$$
I=\alpha_{1} C_{D}+\alpha_{2} \frac{1}{2} \int_{\mathcal{B}}\left(p-p_{d}\right)^{2} d S+\alpha_{3} C_{W},
$$

where $C_{W} \equiv \frac{W}{q_{\infty} S_{r e f}}$ is a dimensionless measure of the wing weight, which can be estimated either from statistical formulas, or from a simple analysis of a representative structure, allowing for failure modes such as panel buckling. The coefficient $\alpha_{2}$ is introduced to provide the designer some control over the pressure distribution, while the relative importance of drag and weight are represented by the coefficients $\alpha_{1}$ and $\alpha_{3}$. 
By varying these it is possible to calculate the Pareto front of designs which have the least weight for a given drag coefficient, or the least drag coefficient for a given weight. The relative importance of these can be estimated from the Breguet range equation;

$$
\begin{aligned}
\frac{\delta R}{R} & =-\left(\frac{\delta C_{D}}{C_{D}}+\frac{1}{\log \frac{W_{1}}{W_{2}}} \frac{\delta W_{2}}{W_{2}}\right) \\
& =-\left(\frac{\delta C_{D}}{C_{D}}+\frac{1}{\log \frac{W_{1}}{W_{2}}} \frac{\delta C_{W}}{\frac{W_{2}}{q_{\infty} S_{r e f}}}\right) .
\end{aligned}
$$

Figure 5 shows the Pareto front obtained from a study of the Boeing 747 wing, ${ }^{36}$ in which the flow was modeled by the Euler equations. The wing planform and section were varied simultaneously, with the planform defined by six parameters; sweepback, span, the chord at three span stations, and wing thickness. The weight was estimated from an analysis of the section thickness required in the structural box. The figure also shows the point on the Pareto front when $\frac{\alpha_{3}}{\alpha_{1}}$ is chosen such that the range of the aircraft is maximized. The optimum wing, as illustrated in figure 6, has a larger span, a lower sweep angle, and a thicker wing section in the inboard part of the wing. The increase in span leads to a reduction in the induced drag, while the section shape changes keep the shock drag low. At the same time the lower sweep angle and thicker wing section reduce the structural weight. Overall, the optimum wing improves both aerodynamic performance and structural weight. The drag coefficient is reduced from 108 counts to 87 counts (19\%), while the weight factor $C_{W}$ is reduced from 455 counts to 450 counts (1\%).

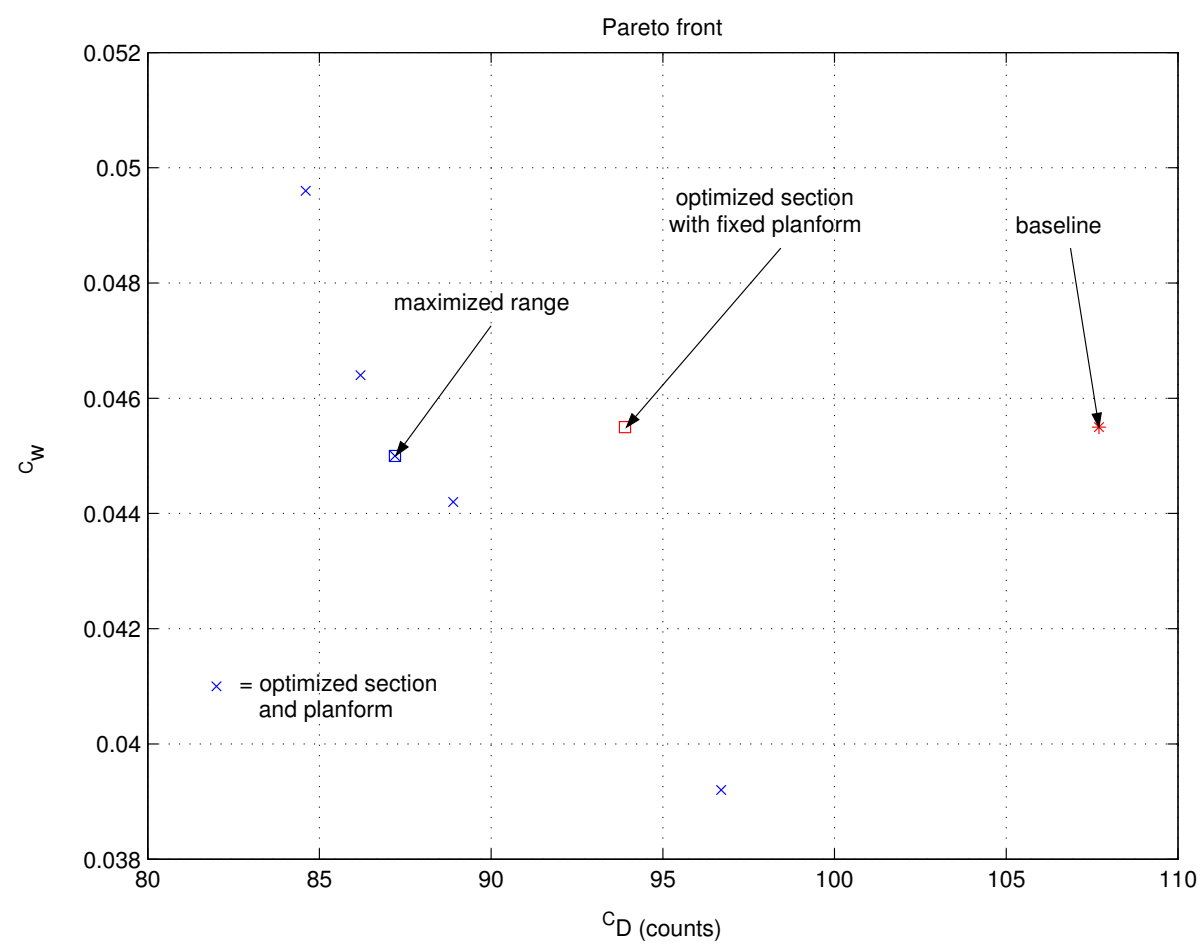

Figure 5. Pareto front of section and planform modifications 


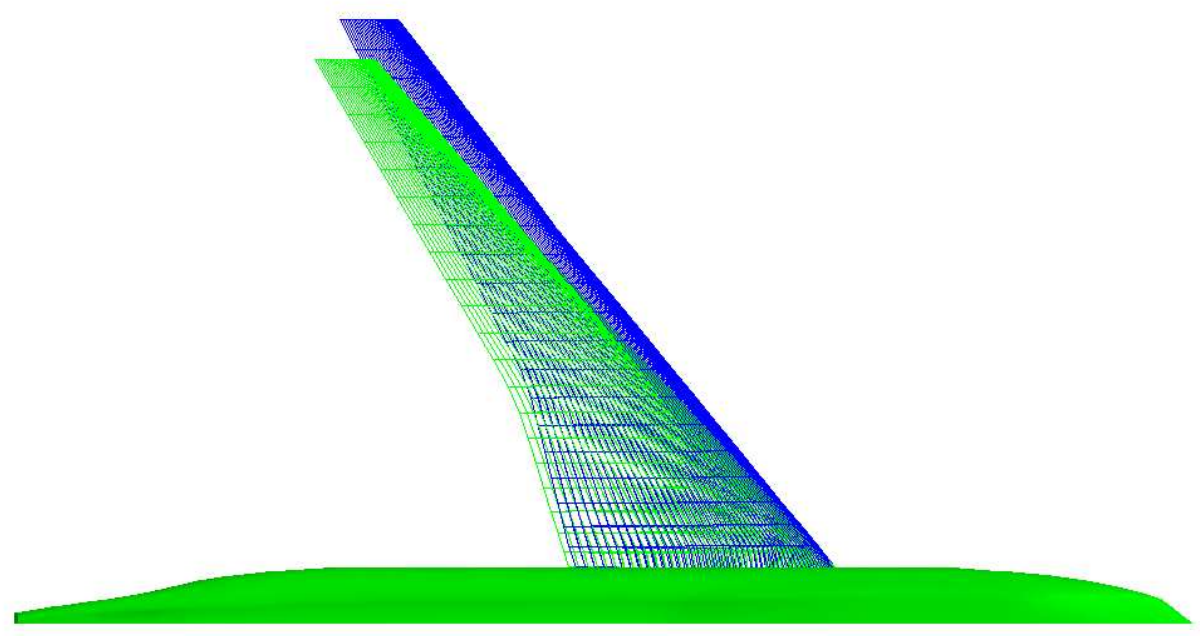

Figure 6. Superposition of the baseline (green) and the optimized section-and-planform (blue) geometries of Boeing 747. The redesigned geometry has a longer span, a lower sweep angle, and thicker wing sections, improving both aerodynamic and structural performances. The optimization is performed at Mach .87 and fixed $C_{L} .42$, where $\frac{\alpha_{3}}{\alpha_{1}}$ is chosen to maximize the range of the aircraft.

\section{Super B747}

In order to explore the limits of attainable performance the B747 wing has been replaced by a completely new wing to produce a "Super B747". An initial design was created by blending supercritical wing sections obtained from other optimizations to the optimum planform which was found in the planform study described in the previous section. Then the RANS optimization code Syn107 was used to obtain minimize drag over 3 design points at Mach $.78, .85$, and .87 , shown in figures 7 (a)-(c) with a fixed lift coefficient of .45 for the exposed wing, corresponding to a lift coefficient of about .52 when the fuselage lift is included. Because the new wing sections are significantly thicker, the new wing is estimated to be 12,000 pounds lighter than the baseline B747 wing as shown in table 1. At the same time the drag is reduced over the entire range from Mach .78 to .90 with a maximum benefit of 25 counts at Mach .87 , as shown in figure 7 (d). Figure 8 and table 2 display the lift-drag polar at Mach .86. The drag coefficient of the Super B747 is 142 counts at a lift coefficient of .5, whereas the baseline B747 has the same drag at a lift coefficient of .45. This represents improvement in $L / D$ of more than 10 percent. In combination with the reduction in wing weight and an increase in fuel volume due to the thicker wing section, this should lead to an increase in range which is substantially more than 10 percent.

Table 1. Comparison between Baseline B747 and Super B747 at Mach .86

\begin{tabular}{|c|c|c|c|}
\hline & $C_{L}$ & $\begin{array}{c}C_{D} \\
\text { counts }\end{array}$ & $\begin{array}{c}C_{W} \\
\text { counts }\end{array}$ \\
\hline Boeing 747 & .45 & 141.3 & 499 \\
& & $(107.0$ pressure, 34.3 viscous $)$ & $(82,550 \mathrm{lbs})$ \\
\hline Super B747 & .50 & 141.9 & 427 \\
& & $(104.8$ pressure, 37.1 viscous $)$ & $(70,620 \mathrm{lbs})$ \\
\hline
\end{tabular}




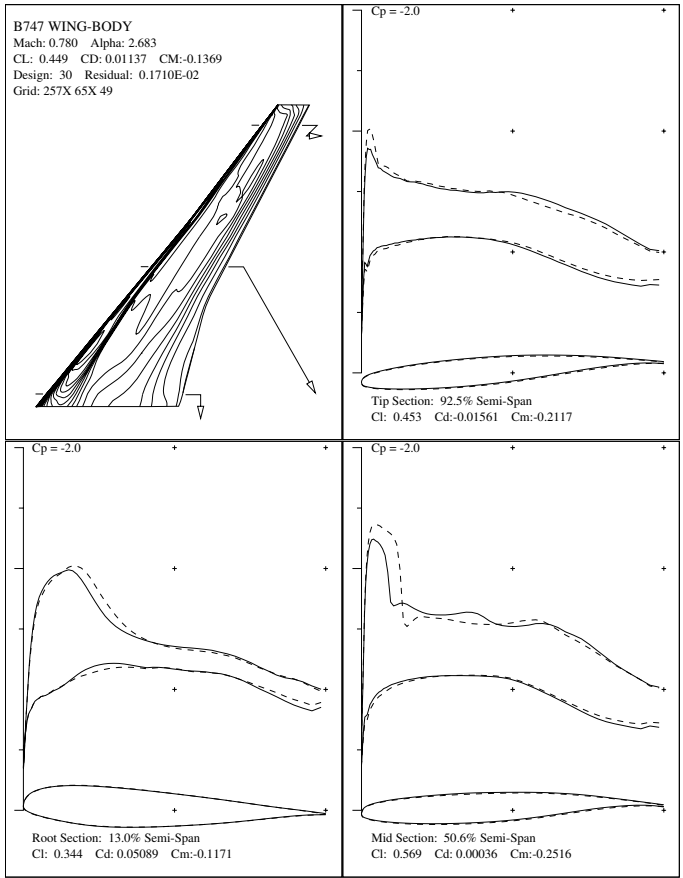

(a) Mach .78

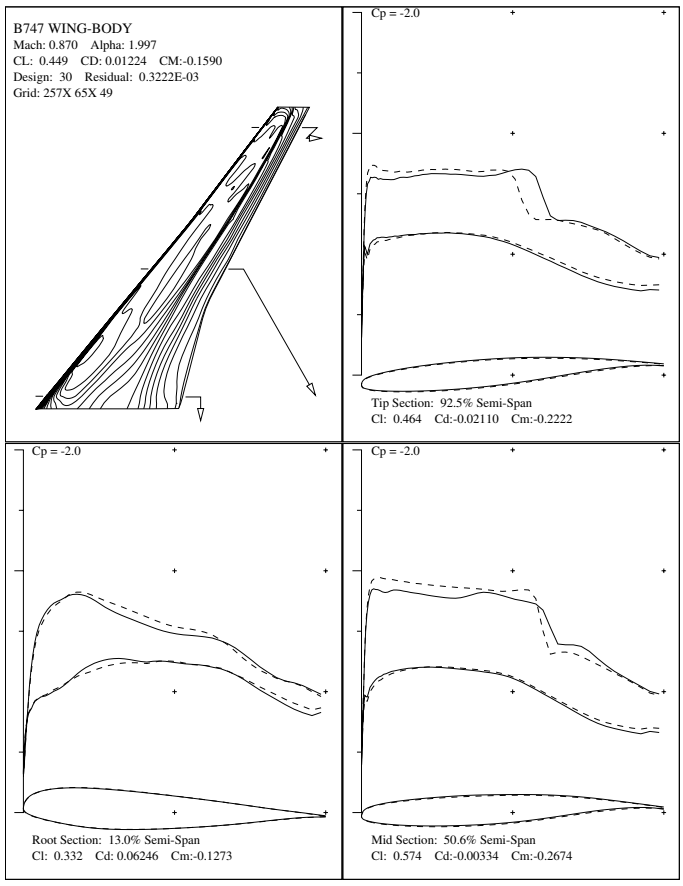

(c) Mach .87

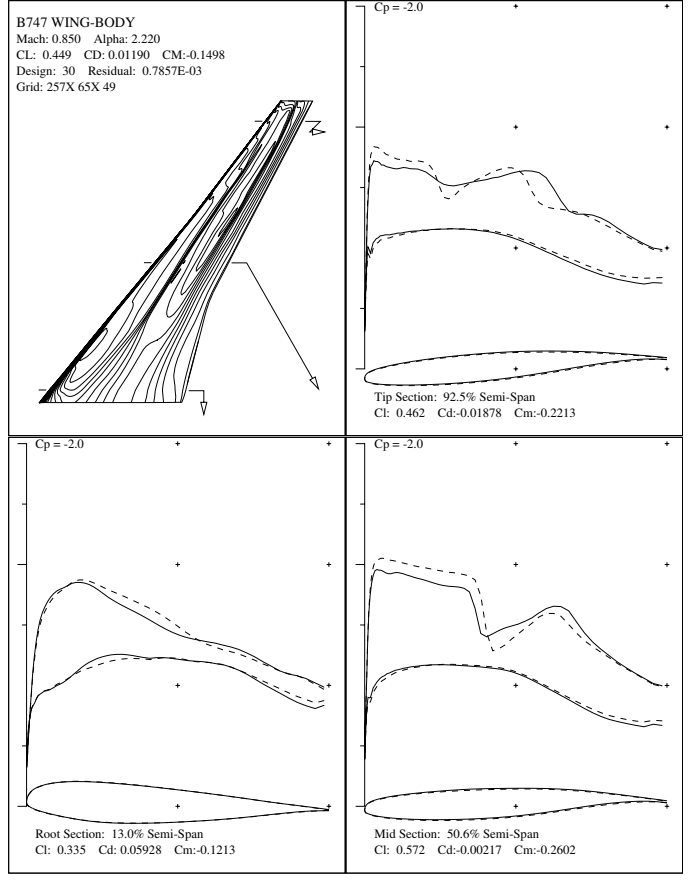

(b) Mach .85

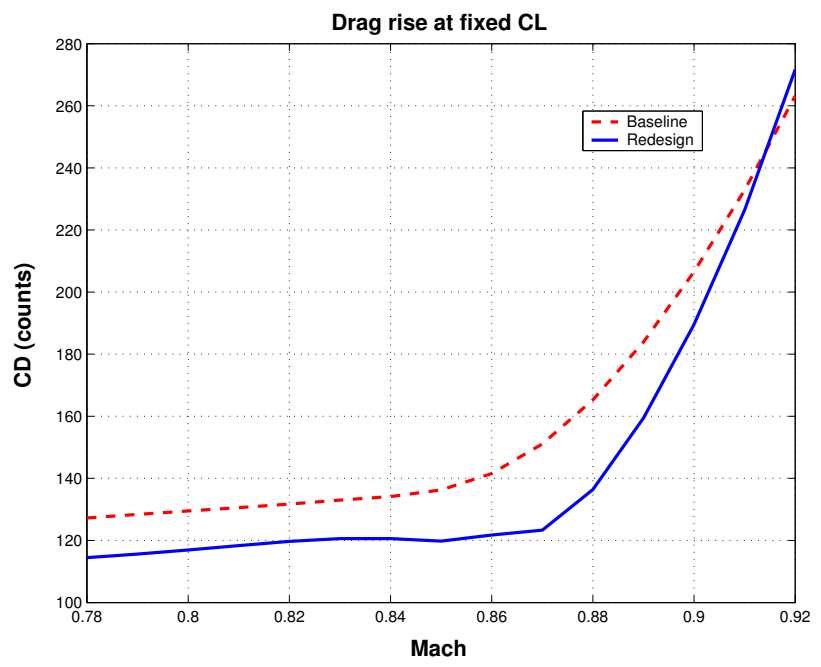

(d) Drag Vs. Mach number

Figure 7. (a)-(c): Super B747 at Mach $.78, .85$, and .87 respectively. Dash line represents shape and pressure distribution of the initial configuration. Solid line represents those of the redesigned configuration. (d): Drag Vs. Mach number of Super B747. 


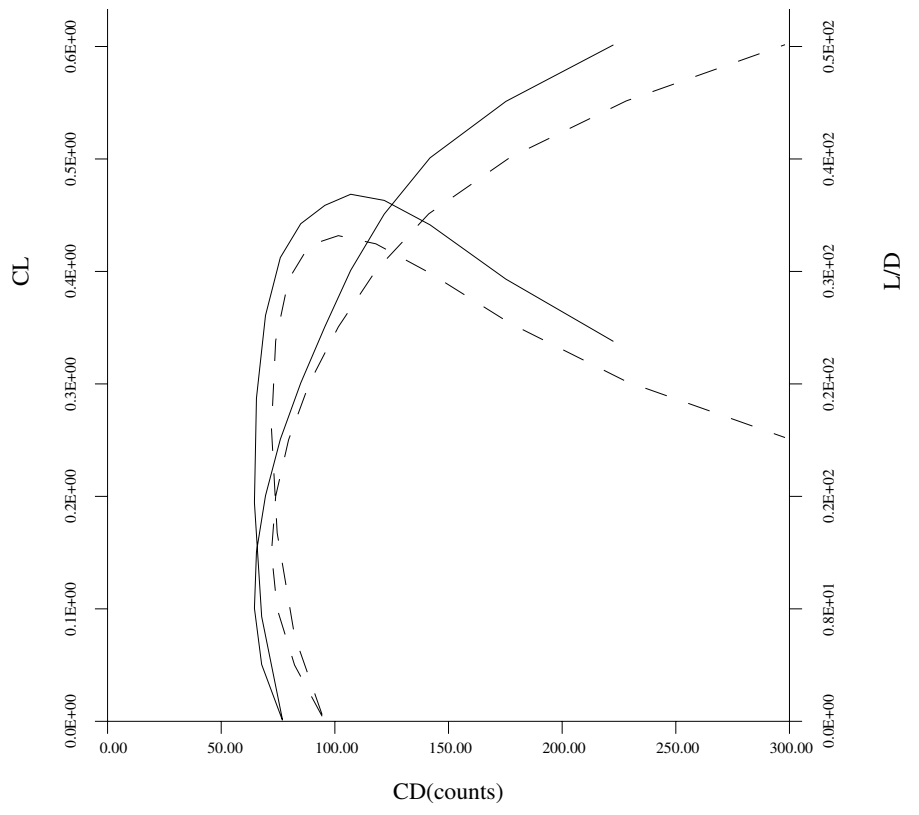

Figure 8. Drag Polars of Baseline and Super B747 at Mach .86. (Solid-line represents Super B747. Dash-line represents Baseline B747.)

Table 2. Comparison of drag polar; B747 Vs. Super B747

\begin{tabular}{rr|rr}
\hline \multicolumn{2}{c|}{ Boeing 747} & \multicolumn{2}{c}{ Super $\mathrm{B} 747$} \\
$C_{L}$ & \multicolumn{1}{c|}{$C_{D}$} & \multicolumn{1}{c}{$C_{L}$} & \multicolumn{1}{c}{$C_{D}$} \\
\hline 0.0045 & 94.3970 & 0.0009 & 76.9489 \\
0.0500 & 82.2739 & 0.0505 & 67.8010 \\
0.1000 & 74.6195 & 0.1005 & 64.6147 \\
0.1501 & 72.1087 & 0.1506 & 65.5073 \\
0.2002 & 73.9661 & 0.2006 & 69.4840 \\
0.2503 & 79.6424 & 0.2507 & 76.0041 \\
0.3005 & 88.7551 & 0.3008 & 84.9889 \\
0.3507 & 101.5293 & 0.3509 & 95.6117 \\
0.4009 & 118.0487 & 0.4010 & 106.9625 \\
$\mathbf{0 . 4 5 1 2}$ & $\mathbf{1 4 1 . 2 9 2 7}$ & 0.4510 & 121.7183 \\
0.5014 & 177.0959 & $\mathbf{0 . 5 0 1 0}$ & $\mathbf{1 4 1 . 8 6 7 5}$ \\
0.5516 & 228.1786 & 0.5512 & 175.2569 \\
0.6016 & 298.0458 & 0.6014 & 222.5459 \\
\hline
\end{tabular}

$\left(C_{D}\right.$ in counts $)$

Note equal drag of the baseline B747 at $C_{L} .45$ and the Super B747 at $C_{L} .5$. 


\section{Shape optimization for a transonic business Jet}

The unstructured design method has also been applied to several complete aircraft configurations. The results for a business jet are shown in figures 9 (a) and (b). There is a strong shock over the out board wing sections of the initial configuration, which is essentially eliminated by the redesign. The drag was reduced from 235 counts to 215 counts in about 8 design cycles. The lift was constrained at 0.4 by perturbing the angle of attack. Further, the original thickness of the wing was maintained during the design process ensuring that fuel volume and structural integrity will be maintained by the redesigned shape. Thickness constraints on the wing were imposed on cutting planes along the span of the wing and by transferring the constrained shape movement back to the nodes of the surface triangulation.

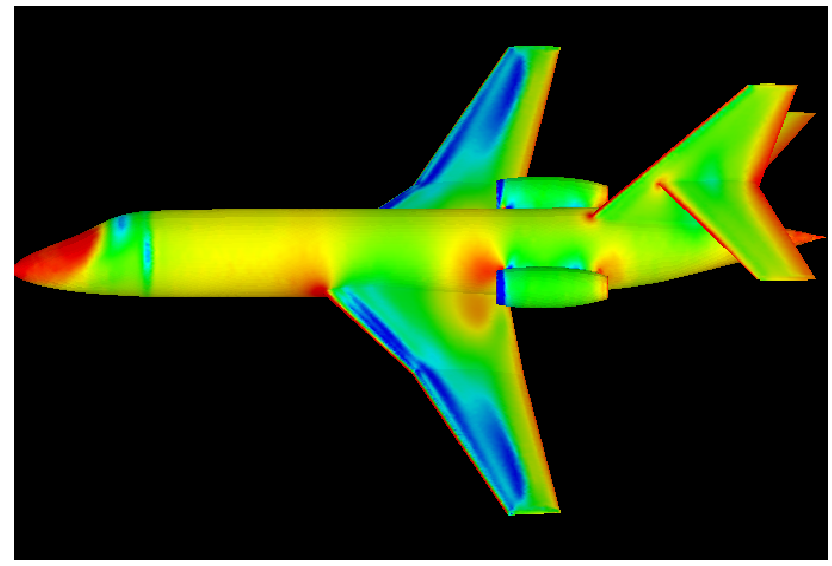

(a) Baseline

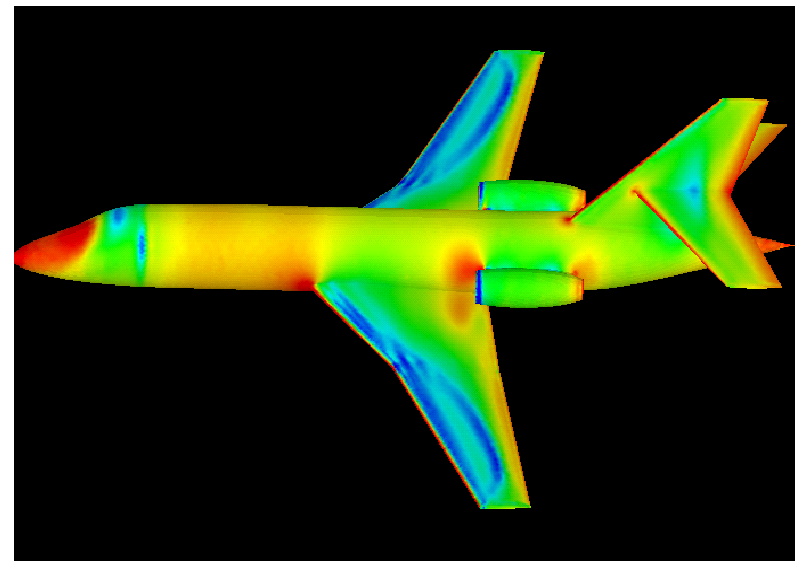

(b) Redesign

Figure 9. Density contours for a business jet at $M=0.8, \alpha=2^{\circ}$

\section{Conclusion}

An important conclusion of both the two- and the three-dimensional design studies is that the wing sections needed to reduce shock strength or produce shock-free flow do not need to resemble the familiar flat-topped and aft-loaded super-critical profiles. The section of almost any of the aircraft flying today, such as the Boeing 747 or McDonnell-Douglas MD 11, can be adjusted to produce shock-free flow at a chosen design point. The accumulated experience of the last decade suggests that most existing aircraft which cruise at transonic speeds are amenable to a drag reduction of the order of 3 to 5 percent, or an increase in the drag rise Mach number of at least .02. These improvements can be achieved by very small shape modifications, which are too subtle to allow their determination by trial and error methods. When larger scale modifications such as planform variations or new wing sections are allowed, larger gains in the range of 5-10 percent are attainable. The potential economic benefits are substantial, considering the fuel costs of the entire airline fleet. Moreover, if one were to take full advantage of the increase in the lift to drag ratio during the design process, a smaller aircraft could be designed to perform the same task, with consequent further cost reductions. Methods of this type will surely provide a basis for aerodynamic designs of the future.

\section{Acknowledgement}

The research described in this paper has benefited tremensously from the continuing support of the Air Force Office of Science Research under grant No. AF F49620-98-1-2004. since 1990.

\section{References}

${ }^{1}$ Godunov, S., "A Difference Method for the Numerical Calculation of Discontinuous Solutions of Hydrodynamic Equations," Mat. Sbornik, Vol. 47, 1959, pp. 271-306, Translated as JPRS 7225 by U.S. Dept. of Commerce, 1960.

${ }^{2}$ Rizzi, A. and Viviand, H., "Numerical Methods for the Computation of Inviscid Transonic Flows with Shock Waves," Proc. GAMM Workshop, Stockholm, 1979.

${ }^{3}$ Jameson, A. and Caughey, D. A., "How Many Steps are Required to Solve the Euler Equations of Steady Compressible Flow: In Search of a Fast Solution Algorithm," AIAA paper 2001-2673, 15th AIAA Computational Fluid Dynamics Conference, Anaheim, California, June 11-14 2001. 
${ }^{4}$ Jameson, A., "Aedynamics," Encyclopedia of Computational Mechanics, edited by E. Stein, R. de Borst, and T. J. Hughes, chap. 11, John Wiley \& Son, 2004, ISBN:0-470-84699-2.

${ }^{5}$ Thwaites, B., editor, Incompressible Aerodynamic, Oxford University Press, 1960, Translated by S.K. Mitter.

${ }^{6}$ Hicks, R. M. and Henne, P. A., "Wing Design by Numerical Optimization," AIAA paper 79-0080, 1979.

${ }^{7}$ Pironneau, O., Optimal Shape Design for Elliptic Systems, Springer-Verlag, New York, 1984.

${ }^{8}$ Lions, J. L., Optimal Control of Systems Governed by Partial Differential Equations, Springer-Verlag, New York, 1971, Translated by S.K. Mitter.

${ }^{9}$ Jameson, A., "Aerodynamic Design via Control Theory," Journal of Scientific Computing, Vol. 3, 1988, pp. 233-260.

${ }^{10}$ Jameson, A., "Computational Aerodynamics for Aircraft Design," Science, Vol. 245, July 1989, pp. 361-371.

${ }^{11}$ Nadarajah, S. K., The Discrete Adjoint Approach to Aerodynamic Shape Optimization, Ph.d. dissertation, Department of Aeronautics and Astronautics, Stanford University, Stanford, CA, January 2003.

${ }^{12}$ Reuther, J., "Aerodynamic Shape Optimization Using Control Theory," Ph. D. Dissertation, University of California, Davis, Davis, CA, June 1996.

${ }^{13}$ Reuther, J., Alonso, J., Rimlinger, M., and Jameson, A., "Aerodynamic Shape Optimization of Supersonic Aircraft Configurations via an Adjoint Formulation on Parallel Computers," AIAA paper 96-4045, 6th AIAA/NASA/ISSMO Symposium on Multidisciplinary Analysis and Optimization, Bellevue, WA, September 1996.

${ }^{14}$ Jameson, A., Schmidt, W., and Turkel, E., "Numerical Solutions of the Euler Equations by Finite Volume Methods with Runge-Kutta Time Stepping Schemes," AIAA paper 81-1259, January 1981.

${ }^{15}$ Jameson, A. and Baker, T., "Improvements to the Aircraft Euler Method," AIAA paper 87-0452, AIAA 25th Aerospace Sciences Meeting, Reno, Nevada, January 1987.

${ }^{16}$ Barth, T. J., "Apects of unstructured Grids and Finite Volume Solvers for the Euler and Navier Stokes Equations," AIAA paper 91-0237, AIAA Aerospace Sciences Meeting, Reno, NV, January 1991.

${ }^{17}$ Leoviriyakit, K. and Jameson, A., "Aerodynamic Shape Optimization of Wings including Planform Variations," AIAA paper 2003-0210, 41 ${ }^{\text {st }}$ Aerospace Sciences Meeting \& Exhibit, Reno, Nevada, January 2003.

${ }^{18}$ Jameson, A., "A perspective on computational algorithms for aerodynamic analysis and design," Progress in Aerospace Sciences, Vol. 37, 2001, pp. 197-243.

${ }^{19} \mathrm{Kim}, \mathrm{S}$., Alonso, J. J., and Jameson, A., "Design Optimization of High-Lift Configurations Using a Viscous Continuous Adjoint Method," AIAA paper 2002-0844, AIAA 40th Aerospace Sciences Meeting \& Exhibit, Reno, NV, January 2002.

${ }^{20}$ Leoviriyakit, K., Kim, S., and Jameson, A., "Viscous Aerodynamic Shape Optimization of Wings including Planform Variables," AIAA paper 2003-3498, 21 ${ }^{\text {st }}$ Applied Aerodynamics Conference, Orlando, Florida, June 23-26 2003.

${ }^{21}$ Jameson, A., Sriram, and Martinelli, L., "An unstructured adjoint method for transonic flows," AIAA paper, $16^{\text {th }}$ AIAA CFD Conference, Orlando, FL, June 2003.

${ }^{22}$ Hicks, R. M. and Henne, P. A., "Wing Design by Numerical Optimization," Journal of Aircraft, Vol. 15, 1978, pp. 407412.

${ }^{23}$ Bischof, C., Carle, A., Corliss, G., Griewank, A., and Hovland, P., "Generating derivative codes from Fortran programs," Internal report MCS-P263-0991, Computer Science Division, Argonne National Laboratory and Center of Research on Parallel Computation, Rice University, 1991.

${ }^{24}$ Green, L. L., Newman, P. A., and Haigler, K. J., "Sensitivity derivatives for advanced CFD algorithm and viscous modeling parameters via automatic differentiation," AIAA paper 93-3321, 11th AIAA Computational Fluid Dynamics Conference, Orlando, Florida, 1993.

${ }^{25}$ Anderson, W. K., Newman, J. C., Whitfield, D. L., and Nielsen, E. J., "Sensitivity Analysis for the Navier-Stokes Equations on Unstructured Meshes using Complex Variables," AIAA paper 99-3294, Norfolk, VA, June 1999.

${ }^{26}$ Jameson, A., Martinelli, L., Alonso, J. J., Vassberg, J. C., and Reuther, J., "Simulation Based Aerodynamic Design," IEEE Aerospace Conference, Big Sky, MO, March 2000.

${ }^{27}$ Jameson, A., "Optimum Aerodynamic Design using Control Theory," Computational Fluid Dynamics Review, 1995 , pp. $495-528$.

${ }^{28}$ Jameson, A., "Optimum Aerodynamic Design Using CFD and Control Theory," AIAA paper 95-1729, AIAA 12th Computational Fluid Dynamics Conference, San Diego, CA, June 1995.

${ }^{29}$ Jameson, A., Martinelli, L., and Pierce, N. A., "Optimum Aerodynamic Design Using the Navier-Stokes Equations," Theoret. Comput. Fluid Dynamics, Vol. 10, 1998, pp. 213-237.

${ }^{30}$ Jameson, A. and Martinelli, L., "Aerodynamic Shape Optimization Techniques Based on Control Theory," Lecture notes in mathematics \#1739, proceeding of computational mathematics driven by industrial problems, CIME (International Mathematical Summer (Center), Martina Franca, Italy, June 21-27 1999.

${ }^{31}$ Jameson, A., "Aerodynamic Shape Optimization Using the Adjoint Method," 2002-2003 lecture series at the von karman institute, Von Karman Institute For Fluid Dynamics, Brussels, Belgium, Febuary 3-7 2003.

32 Jameson, A., Martinelli, L., and Vassberg, J., "Reduction of the Adjoint Gradient Formula in the Continuous Limit," AIAA paper, $41^{\text {st }}$ AIAA Aerospace Sciences Meeting, Reno, NV, January 2003.

${ }^{33}$ Martinelli, L. and Jameson, A., "Validation of a Multigrid Method for the Reynolds Averaged Equations," AIAA paper 88$0414,1988$.

${ }^{34}$ Tatsumi, S., Martinelli, L., and Jameson, A., "A new High Resolution Scheme for Compressible Viscous Flows with Shocks," AIAA paper To Appear, AIAA 33nd Aerospace Sciences Meeting, Reno, Nevada, January 1995.

${ }^{35}$ Harbeck, M., "Exploring the Limits of Shock-free Transonic Airfoil Design," Problems in Aeronautics and Astronautics Report, Stanford University, Stanford, CA, July 2004.

${ }^{36}$ Leoviriyakit, K. and Jameson, A., "Aero-Structural Wing Planform Optimization," Proceedings of the $42^{\text {st }}$ Aerospace Sciences Meeting \& Exhibit, Reno, Nevada, January 2004. 